\title{
Interplay of transcription factors STAT3, STAT1 and AP-1 mediates activity of the matrix metallo-proteinase-1 promoter in colorectal carcinoma cells
}

\author{
A. MÜLLER ${ }^{1,2}$, J. GASCH${ }^{1,3}$, K. F. ALBRING ${ }^{1,4}$, F. ABERGER ${ }^{5}$, H. NIVARTHI $^{6,7}$, M. KHEMERI ${ }^{8}$, R. MORIGGL ${ }^{6}$, K. H. FRIEDRICH ${ }^{1, \star}$
}

${ }^{1}$ Institute of Biochemistry II, Jena University Hospital, Jena, Germany; ${ }^{2}$ Clinic and Polyclinic of Neurology, University Hospital Leipzig, Leipzig, Germany; ${ }^{3}$ Helios-Klinikum Erfurt, Erfurt, Germany; ${ }^{4}$ Carl-von-Ossietzky-Gymnasium, Berlin, Germany; ${ }^{5}$ Department of Molecular Biology, University of Salzburg, Salzburg, Austria; ${ }^{6}$ Ludwig Boltzmann Institute for Cancer Research, Medical University of Vienna, Vienna, Austria; ${ }^{7}$ Research Center for Molecular Medicine of the Austrian Academy of Sciences, Vienna, Austria; ${ }^{8}$ Faculty of Sciences of Bizerte, IMEC, Bizerte, Zarzouna, Tunisia

*Correspondence: karlheinz.friedrich@med.uni-jena.de

Received July 31, 2018 / Accepted November 6, 2018

\begin{abstract}
Signal Transducers (STATs) 1 and 3 and Activator Protein 1 (AP-1) are transcription factors involved in the development of malignancy in colorectal carcinoma (CRC). Matrix Metalloproteinase 1 (MMP-1) is a protease frequently dysregulated in de-differentiated and invasive cancer cells. Its expression is influenced by STAT and AP-1 transcription factors. We studied their contributions to transcriptional regulation of MMP-1 in colorectal carcinoma (CRC) cells. Both STAT3 and AP-1 have individual expression-inducing and additive effects and interact with the MMP-1 promoter. DNA binding of AP-1 protein c-Jun is stimulation-independent, but modulated by STAT3 and a STAT recognition DNA element. Activated STAT3 showed a suppressive effect on AP-1-mediated MMP-1 mRNA upregulation as shown by STAT3 knock-down. Surprisingly, activated STAT1 overcame STAT3-dependent repression of AP-1-driven MMP-1 expression. Moreover, combined STAT3, STAT1 and AP-1 activities evoked maximal MMP-1 mRNA levels in a synergistic manner. Our results suggest a dominant role of AP-1 in transcriptional upregulation of MMP-1 in CRC cells which is modulated by joint functions of STAT3 and STAT1. The individual and combinatorial activity of these factors is of diagnostic and prognostic interest.
\end{abstract}

Key words: colorectal cancer, transcription factors, transcriptional regulation, matrix metalloproteinase

Colorectal carcinoma (CRC) is one of the most frequent malignant diseases with a mortality rate close to $50 \%$ [1]. It is, hence, a prime subject of molecular cancer research which revealed crucial roles of dysregulated signalling via Wnt and Ras-Raf pathways [2]. Signalling reactions resulting in altered transcriptional regulation through nuclear factor $\mathrm{\kappa B}$ $(\mathrm{NF} \kappa \mathrm{B})$ and Activator protein 1 (AP-1) have been implicated in cancer-connected inflammation and development of the metastatic phenotype [3]. Importantly, inadequate signal transduction by cytokine driven Janus kinases (JAKs) and signal transducers and activators of transcription (STATs) has also been associated with the development and progression of CRC [4].

Aberrant STAT3 tyrosine phosphorylation and DNA binding potential was observed in the majority of CRC tissues, and experiments with CRC cell lines revealed important functions of activated STAT3 in the control of malignancy parameters such as cell proliferation, motility and invasiveness $[4,5]$. Hyper-activation of STAT3 in CRC tissue as well as in CRC cell lines is statistically and functionally connected with enhanced expression of malignancy-associated matrix metalloproteinase-1 (MMP-1) [6]. While STAT3 activity is mostly regarded as a driver of enhanced malignancy and invasiveness of CRC cells [5-8], STAT1 is known for tumor suppressive functions and association with favorable prognosis in various cancers $[9,10]$.

MMP-1 is an interstitial collagenase with importance for invasive properties of malignant tumors $[11,12]$ and associated with poor prognosis of CRC patients [13, 14] and, notably, with STAT3 activity in CRC tissue $[6,15]$. Recently we have studied the contribution of STAT3 and AP-1 to transcriptional control human MMP-1 promoter in CRC cells and showed that the two transcriptional factors cooperate through direct and indirect interaction with a combined binding element immediately upstream of the transcriptional start site. We obtained evidence for an important role of protein-protein contacts between STAT3 and c-Jun for MMP-1 gene regulation and, furthermore, observed clear coincidence for concerted aberrant activation of both STAT3 and AP-1 in human colon cancer biopsies [16]. 
While STAT3, on one hand, was regarded as a negative predictor, STAT1, on the other hand, was considered a favorable mediator for the outcome of solid cancers in general and of CRC in particular. Our recent results have shattered the simplistic view of opposing influences of STAT3 and STAT1 on the CRC development. We rather obtained evidence from both human CRC biopsies and from xenograft experiments in mice that the ratio of STAT3 and STAT1 activity and expression is relevant for the progression of CRC $[17,18]$. Since the mechanistic basis of these findings is yet not understood, we used the well characterized MMP-1 promoter as a model to study the individual and combined effects of STAT3/1 and AP-1 on transcriptional regulation of a gene with relevance for CRC.

\section{Material and methods}

Cells and DNA constructs. Colon carcinoma cell line HT-29 was obtained from the American Type Culture Collection (ATCC). Cells were grown in RPMI 1640 medium containing $10 \%$ fetal calf serum, $200 \mathrm{mM}$ L-glutamine, $100 \mathrm{mM}$ sodium pyruvate and $1 \mathrm{mg} / \mathrm{ml}$ gentamycin in coated tissue culture plastic-ware (Greiner Labortechnik, Frickenhausen, Germany).

The MMP-1 promoter/luciferase reporter plasmid -4272 hMMP-luci (GenBank Accession Number AF023338), obtained from C. E. Brinckerhoff (Dartmouth Medical School, Hanover, New Hampshire) was described previously [19].

Promoter deletion construct hMMP $\Delta \mathrm{Mlu} / \mathrm{Sph}$ was generated by replacing a $305 \mathrm{bp}$ Mlu I/Sph I fragment of -4272 hMMP-luci comprising the STAT cognate element MMP 1A with a synthetic Eco RI linker. The Mlu I site originated from the multiple cloning site of pGL3 luc, the Sph I site was located at position -3985 of the MMP-1 promoter. The Eco RI linker was obtained by hybridization of oligonucleotides pGLMluSphup (5'-CGCGTCGTCGAATTCTCACGCATG-3') and pGLMluSphlo (5'-CGTGAGAATTCGACGA-3'). The shortened promoter MMP-1 promoter construct hMMP-1 $-0.6 \mathrm{~kb}$ was generated by complete digestion of -4272 hMMP-luci with Kpn I and religation of the plasmid backbone, resulting in the loss of $3.8 \mathrm{~kb}$ distal promoter sequence. Variant derivatives of pMMP- $1-0.6 \mathrm{~kb}$ with mutations in the STAT binding element at position -114 or/and the AP- 1 binding element at -133 were generated by replacing the Kpn I/ Hind III fragment of pMMP-1 $-0.6 \mathrm{~kb}$ with mutated fragments obtained by recombinant PCR and subsequent Kpn I/Hind III cleavage. Flanking primers used were pGLKpnPCRup (5'-GCAATAGGGTACCAGGCAGCTTAACAAAGG-3') and pMMP1HindPCRlo (5'-CGGAATGCCAAGCTTACTTAGATCGCAGAT-3'), mutagenic primers were STAT3BEmutup (5'-GTCAGACACCTCTGGCTTTCTATCAGGGCAAGGACTCT-3') and STAT3BEmutlo (5'-CTTGCCCTGATAGAAAGCCAGAGGTGTC-3') for the mutation in the STAT3 binding element and AP1BEmutup (5'-GGATGTTATAAAGCTAGAGTCAGACACCTCTGGC-3') AP1BEmutlo (5'-AGAAAGCCAGAGGTGTCTGACTCTAGCTTTATAAC-3') for the mutation in the AP-1 binding element.

Transient knockdown of STAT3 in HT-29 cells was performed by transfection as described above with plasmid pSUPERneo encoding the STAT3-specific RNAi sequence 5'-GATCCCCTTCAGACCCGTCAACAAATTCAAGAGATTTGTTGACGGGTCTGAAGTTTTT-3' (pSUPERneoSTAT3si) or a corresponding scrambled (scr) sequence (pSUPERneoSTAT3scr) [20], or, as control with empty pSUPERneo vector. Efficiency and specificity of STAT3 depletion was verified $96 \mathrm{~h}$ post transfection by Western blot detection using an anti-STAT3 antibody.

STAT3 knockdown in HT-29 cells was achieved by expression of the lentiviral construct shSTAT3 TRCN0000071456 from the Sigma MISSION shRNA library (Sigma-Aldrich, Taufkirchen, Germany) or a control scrambled shRNA (SHC002), respectively, as described previously [17].

Luciferase reporter gene assay. $7 \times 10^{5}$ exponentially growing HT-29 cells and derivatives were plated in six-well cluster plates (Greiner) in $2 \mathrm{ml}$ RPMI 1640/10\% FCS and grown to $80-90 \%$ confluency. The cells were washed, transferred into $1 \mathrm{ml} /$ well fresh medium, and co-transfected with $1 \mu \mathrm{g}$ of MMP-1 promoter/luciferase construct (wild type or mutant) and $0.1 \mu \mathrm{g}$ of the pRL-TK Renilla luciferase plasmid (Promega, Madison, WI, USA) as an internal control for transfection efficiency by using Polyfect reagent (Qiagen, Hilden, Germany). Both DNAs were diluted with growth medium containing no serum, antibiotics or proteins to a final volume of $100 \mu \mathrm{l}$ and then the solutions were mixed and $9 \mu \mathrm{l}$ of PolyFect per well was added. The mixture was incubated at room temperature for 5-10 $\mathrm{min}$ to allow complex formation before $1 \mathrm{ml}$ medium was added. After 14 h incubation at $37^{\circ} \mathrm{C}$, cells were gently washed with RPMI 1640 and incubated with fresh RPMI, optionally containing $10 \mathrm{ng} / \mathrm{ml}$ Interleukin-6 (IL-6), or/and $100 \mathrm{nM}$ Tetradecanoylphorbol (TPA) and/or $100 \mathrm{ng} / \mathrm{ml}$ interferon- $\gamma$ (IFN- $\gamma$ ). The cells were harvested $8 \mathrm{~h}$ later using "reporter lysis buffer" (Promega, Madison, WI, USA). Luciferase- and Renilla activities were determined using the Dual-Luciferase Reporter Assay System kit from Promega following the manufacturer's instructions. Luminescence was measured using a Micro Luminat LB 96 P Luminometer and reported as relative light units. Three independent transfections, each run in triplicate, were performed and the results were normalized to the Renilla activity.

Western blot. For factor stimulation, cells were incubated with $20 \mathrm{ng} / \mathrm{ml}$ recombinant human IL-6 (R\&D Systems $\mathrm{GmbH}$, Wiesbaden, Germany) for $30 \mathrm{~min}$ (Western blot analysis) or $18 \mathrm{~h}$ (reporter gene assay). Cells were harvested at $80 \%$ confluence after three PBS washes at $4{ }^{\circ} \mathrm{C}$. Whole cell extracts were prepared from cell pellets as described above.

Western blot analysis was performed as described previously [21]. Briefly, cell extracts were solubilized in gel loading 
buffer (62.5 mM Tris/ HCl pH 6.8; 2\% SDS; 25\% glycerol; $1 \%$ phenolblue; $5 \% \beta$-mercaptoethanol), boiled for $10 \mathrm{~min}$ and run through $10 \%$ acrylamide SDS gels. Proteins were blotted onto nitrocellulose membranes (GE Healthcare, Munich, Germany), non-specific binding sites on blot membranes were blocked with $5 \%$ milk protein in Tris buffered saline (TBS)/0.01\% Tween. Primary antibodies directed to tyrosine phosphorylated STAT3, STAT3, tyrosine phosphorylated STAT1, STAT1, c-Jun, GAP-DH and to $\beta$-actin (all purchased from Cell Signaling Technology, Beverly, MA, USA) were used for probing at a 1:1000 dilution. Probing was performed with peroxidase-conjugated anti-rabbit IgG (Roth, Karlsruhe, Germany) at a dilution of 1:10000 followed by visualization using an Enhanced Chemiluminescence detection kit (GE Healthcare).

Removal of bound antibodies from membranes prior to re-probing with other antibodies was achieved by incubation in stripping buffer $(60 \mathrm{mM}$ Tris/ $\mathrm{HCl} \mathrm{pH} \mathrm{6.7;} 2 \%$ SDS; $0.4 \%$ 2-Mercaptoethanol) at $55^{\circ} \mathrm{C}$ for $60 \mathrm{~min}$.

Protein co-immunoprecipitation (Co-IP) and chromatin immunoprecipitation (ChIP). HT-29 cells were grown to $80-90 \%$ confluence, optionally treated with stimuli as described above for $30 \mathrm{~min}$ and then subjected to Co-IP or ChIP.

For Co-IP, roughly $10^{7}$ cells were lysed as described [5]. Aliquots of $200 \mu \mathrm{l}$ cleared lysate were incubated with $2 \mu \mathrm{g}$ of anti-STAT3 pTyr 705 (Cell Signaling Technology) for $30 \mathrm{~min}$, immunocomplexes were collected by centrifugation after binding to $50 \mu$ l protein A sepharose beads (Sigma-Aldrich). Precipitates were analyzed by Western blot detection for phosphorylated STAT3 and c-Jun as described above.

ChIP was performed as described previously [22]. In brief, cells were fixed with $2 \mathrm{mM}$ disuccinimidyl glutarate for $45 \mathrm{~min}$ and cross-linked for $10 \mathrm{~min}$ with $1 \%$ formaldehyde. For immunoprecipitation, $2 \mu \mathrm{g}$ of either anti-STAT3 (His190) (Cell Signaling Technology, Beverly, MA, USA), anti-c-Jun (Cell Signaling) or unspecific IgG (Santa Cruz, Biotechnology, Dallas, TX, USA) as isotype control were employed as indicated. For PCR analysis, $2 \mu \mathrm{l}$ of extracted DNA were used as template and subjected to an initial incubation of $7 \mathrm{~min}$ at $95^{\circ} \mathrm{C}$ followed by 31 cycles of $30 \mathrm{~s}$ at $95^{\circ} \mathrm{C}, 30 \mathrm{~s} 59^{\circ} \mathrm{C}, 10 \mathrm{~s}$ at $72^{\circ} \mathrm{C}$ for $10 \mathrm{~s}$ and a final $2 \mathrm{~min}$ extension step at $72^{\circ} \mathrm{C}$. Products were separated through $8 \%$ polyacrylamide gels. Primers used were MMP-1 promoter forward (5'-GCAGAGTGTGTCTCCTTCGCAC-3') and MMP-1 promoter reverse (5'-AGGTAAGTGATGGCTTCCCAGC-3') binding to positions -217 and +39 relative to the transcriptional start site, respectively.

DNA-protein interaction assay. DNA-protein interactions were monitored by the "ABCD (avidin, biotin, complex, DNA) assay". The protocol based on immobilization of protein-DNA complexes via binding of a biotinylated oligonucleotide to a streptavidin matrix has been described previously [16]. Briefly, $200 \mu \mathrm{l}$ samples of whole cell extract containing roughly $4.5 \mathrm{mg}$ protein $/ \mathrm{ml}$ were incubated for
60 min on ice with $2 \mathrm{mg}$ biotinylated oligonucleotide hybrid dissolved in $200 \mathrm{ml}$ of a buffer containing $100 \mathrm{mM} \mathrm{KCl}$, $20 \mathrm{mM} \mathrm{N}-2$ hydroxyethylpiperazine-N9-2-ethanesulfonic acid (HEPES; pH 7.8), 20\% glycerol, $1 \mathrm{mM}$ dithiothreitol, $0.1 \%$ nonidet $\mathrm{P} 40$, and $10 \mathrm{mg}$ herring sperm DNA. $5 \mu \mathrm{l}$ equilibrated streptavidin agarose beads (Pierce, Rockford, IL, USA) were added and incubation was continued for 30 min at $48^{\circ} \mathrm{C}$ on a rotator. After repeated washing with the buffer described above (except for containing 50 instead of $100 \mathrm{mM} \mathrm{KCl}$ ), beads were boiled in SDS-PAGE sample buffer, and proteins were subjected to Western blot analysis. Biotinylated oligonucleotides were obtained from Metabion $\mathrm{GmbH}$ (Martinsried, Germany), sequences were given in [16]. Hybrids were formed by combining equimolar amounts of respective sense and antisense oligonucleotides in $0.5 \mathrm{M}$ $\mathrm{NaCl}, 0.2 \mathrm{M}$ Tris $\mathrm{pH}$ 7.4, boiling for $2 \mathrm{~min}$ and cooling down to ambient temperature.

RNA extraction, cDNA synthesis and RT-PCR. HT-29 cells were incubated with $10 \mathrm{ng} / \mathrm{ml}$ IL-6 or/and $100 \mathrm{nM}$ TPA for $12 \mathrm{~h}$ and subjected to total RNA isolation using Trizol reagent (Life Technologies, Carlsbad, CA, USA). For cDNA synthesis, Moloney leukemia virus (MLV) reverse transcriptase and random hexamer primers (both from Life Technologies) were used according to the manufacturer's instructions. Quantification of MMP-1 cDNA was performed by real time $\mathrm{PCR}$ employing a $\mathrm{MyiQ}^{\mathrm{TM}}$ instrument (Bio-Rad Laboratories, Munich, Germany) in $25 \mu \mathrm{l}$ reaction volumes containing $12.5 \mu \mathrm{l}$ of Absolute ${ }^{\mathrm{Tm}}$ QPCR SYBR' Green Mix (ABgene, Hamburg, Germany), 0.25 pmol of each primer, and $1 \mu \mathrm{l}$ of cDNA. 40 amplification cycles $\left(30 \mathrm{~s}\right.$ at $94^{\circ} \mathrm{C}, 30 \mathrm{~s}$ at $56^{\circ} \mathrm{C}, 20 \mathrm{~s}$ at $\left.72^{\circ} \mathrm{C}\right)$ were run with an initializing incubation at $95^{\circ} \mathrm{C}$ for $15 \mathrm{~min}$ and a final step at $72^{\circ} \mathrm{C}$ for $1 \mathrm{~min}$. Primers were MMP-1 fw (5'-GGACCAACAATTTCAGAGAGTACA-3'), MMP-1 rv (5'-AGTAGAATGGCAGAGTCCAAGAG-3'), $\beta$-actin sense (5'-GGACTTCGAGCAAGAGATGG-3') and $\beta$-actin antisense (5'-GCAGTGATCTCCTTCTGCATC-3'), the latter two serving as internal control. After establishment of standard curves, the determined copy number of MMP-1 mRNA was normalized to $\beta$-actin mRNA.

\section{Results}

Previously, we demonstrated cooperation of STAT3 and AP-1 in functional control of the MMP-1 promoter [16]. Prompted by the emerging relevance of STAT1 signaling in cancer in general and by our recent findings on non-independent involvement of STAT3 and STAT1 in CRC [23], we extended this study and investigated individual and combined contributions of STAT3, STAT1 and AP-1 to MMP-1 expression in CRC cells. We employed IL-6, TPA and IFN- $\gamma$ as specific stimuli for transcriptional regulation via STAT3, AP-1 and STAT1, respectively, and addressed effects on both MMP-1 promoter activity and MMP-1 mRNA expression in the CRC cell line HT-29. 
STAT3 and c-Jun in DNA-protein and protein-protein interactions on the MMP-1 promoter. We pursued the previously put forward hypothesis that mutual interactions of STAT3 and c-Jun are contributing to their combined influence on transcriptional regulation. We had previously observed that upon IL-6 treatment of cells, pTyr-STAT3 bound to the proximal STAT binding element SBE (for systematic reasons originally named "MMP-1D") and inter-

A
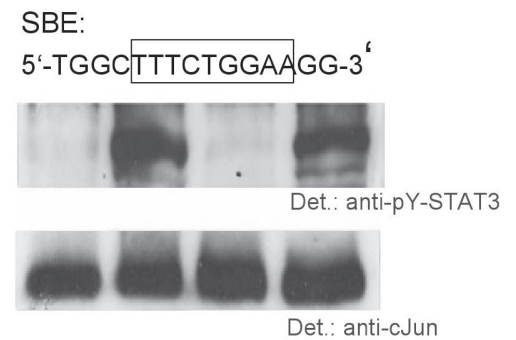

\begin{tabular}{l|c|c|c|c|}
\cline { 2 - 4 } IL-6 & - & + & - & + \\
\cline { 2 - 5 } TPA & - & - & + & + \\
\cline { 2 - 5 } & &
\end{tabular}

\section{B}

$\mathrm{mt}$ SBE: 5'-TGGCTTTCTATCAGG-3"
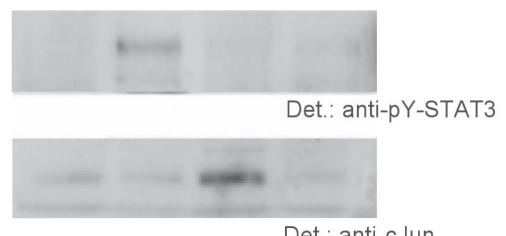

\begin{tabular}{c|c|c|c|c|}
\cline { 2 - 5 } IL-6 & - & + & - & + \\
\cline { 2 - 5 } TPA & - & - & + & + \\
\cline { 2 - 5 } & &
\end{tabular}

estingly, also to the AP-1 BE. Moreover, we detected constitutive binding of c-Jun to the AP-1 BE and surprisingly, also to MMP-1D [16]. Here we extended this analysis by including TPA as a stimulus to active AP-1 signaling alone or in combination with IL-6. Binding of STAT3 and c-jun to binding sites of both wild type and mutated status was assessed (Figures $1 \mathrm{~A}$ and B). In line with previous findings, IL-6 simulation was observed to induce pTyr-STAT3 inter-

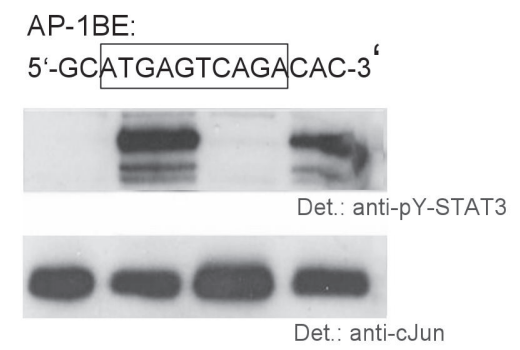

\begin{tabular}{c|c|c|c|c|}
\cline { 2 - 5 } IL-6 & - & + & - & + \\
\cline { 2 - 5 } TPA & - & - & + & + \\
\cline { 2 - 5 } & &
\end{tabular}

mt AP-1BE:
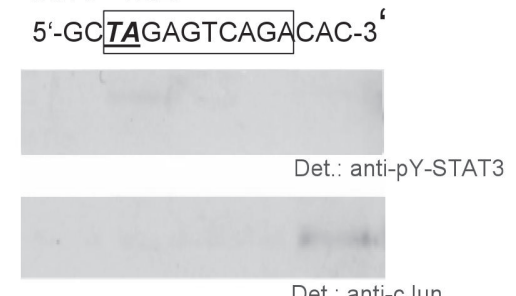

\begin{tabular}{c|c|c|c|c|}
\cline { 2 - 4 } IL-6 & - & + & - & + \\
\cline { 2 - 5 } TPA & - & - & + & + \\
\hline
\end{tabular}

\section{C}

\begin{tabular}{|c|c|c|c|c|c|c|}
\hline Input (10\%) & + & - & - & - & - & \\
\hline IL-6 & - & - & + & - & + & \\
\hline TPA & - & - & - & + & + & \\
\hline & & 25 & $-\infty$ & e & & $\begin{array}{l}\text { IP: anti-pY-STAT3 } \\
\text { Det.: anti-c-Jun }\end{array}$ \\
\hline
\end{tabular}

Figure 1. Analysis of protein-DNA and protein-protein interactions of STAT3 and c-Jun in IL-6 and TPA-stimulated HT-29 cells. A) Stimulationdependent affinities of STAT3 and c-Jun for cognate DNA elements within the twin STAT/AP-1 binding site of the MMP-1 promoter. Samples of DNA double strands formed of biotinylated oligonucleotides containing boxed STAT and AP-1 cognate elements from the human MMP-1 promoter were incubated with lysate from untreated or IL-6 or/and TPA stimulated HT-29 cells as indicated. DNA-protein complexes were collected with streptavidin agarose beads and subjected to Western blot analysis. B) Effects of recognition site disruption for stimulus-dependent affinities of STAT3 and c-Jun for their respective binding elements (SBE and AP-1BE). Double-stranded, biotinylated oligonucleotides containing mutated versions of the boxed proximal SBE and AP-1BE from the proximal section of the MMP-1 promoter (altered nucleotides as compared to the wild-type sequences shown in bold italic) were analyzed for interaction with transcription factors in lysates from stimulated HT-29 cells as in A. C) Analysis of stimulus-induced interactions of STAT3 and c-Jun in HT-29 cells. HT-29 cells were stimulated with IL-6 or/and TPA as indicated, lysed and subjected to immunoprecipitation with an antibody to phospho-STAT3. Precipitates were analyzed by Western blot detection for phosphorylated STAT3 and c-Jun. The leftmost lane represents $10 \%$ of input lysate from untreated cells and serves as a reference. pSTAT3 signals provide an internal control for equivalent amounts of protein subjected to co-precipitation. 
A pMMP-1 - 0,6 kb

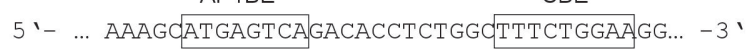

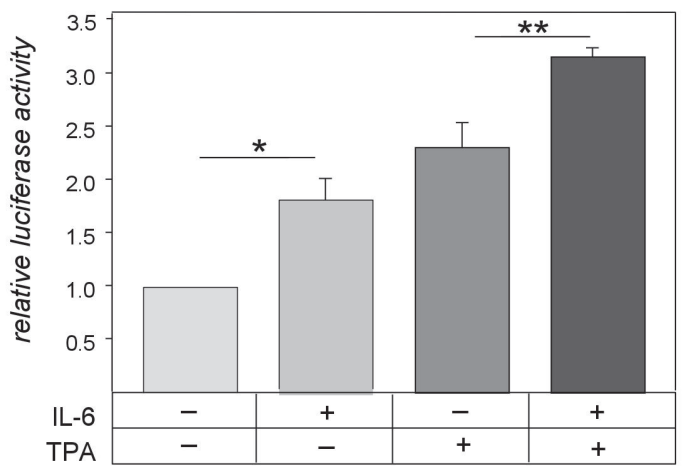

B

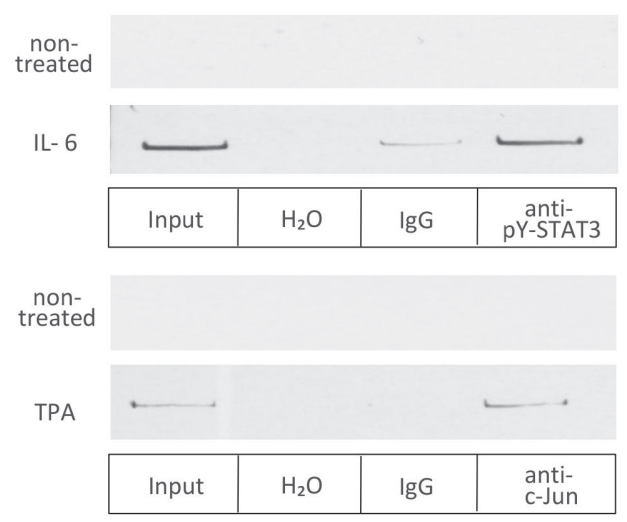

C

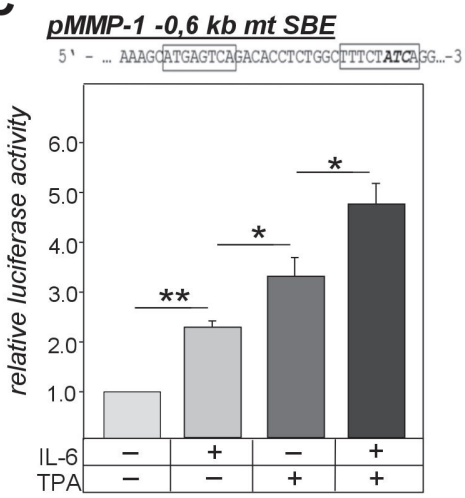

pMMP-1 -0,6 kb mt AP1BE
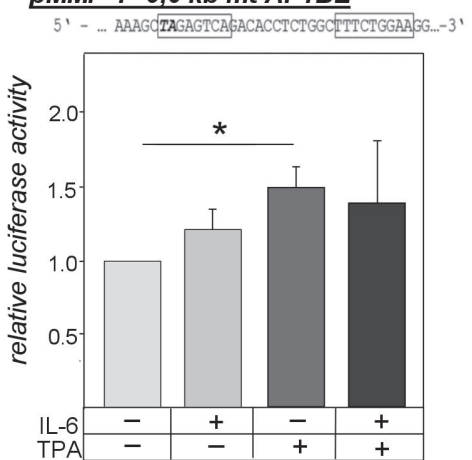

D

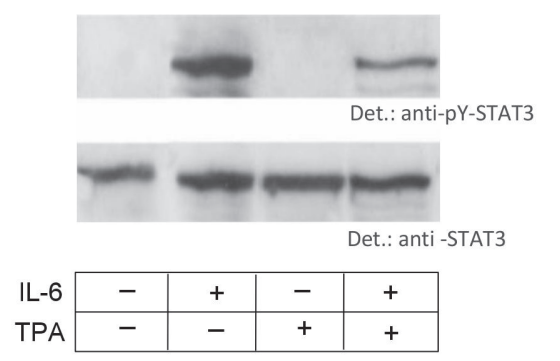

Figure 2. Activities of STAT3 and AP-1 on a minimal human MMP-1 promoter in the CRC cell line HT-29. A) Effect of IL-6- or/and TPA stimulation of reporter gene construct pMMP-1 $-0.6 \mathrm{~kb}$. In the reporter gene plasmid, $0.6 \mathrm{~kb}$ of the MMP-1 promoter sequence upstream of the transcriptional start site are fused to the luciferase coding region [16]. The sequence around the twin STAT/AP-1 biding element (SBE and AP1BE) at position -53 is shown with the consensus cognate sequences boxed in. Cells were co-transfected with reporter gene plasmid along with pRL-TK Renilla luciferase plasmid. $14 \mathrm{~h}$ post transfection, cells were stimulated with IL-6 or/and $100 \mathrm{nM}$ TPA as indicated and analyzed for Luciferase- and Renilla activities (expressed as relative light units normalized to Renilla activity). Three independent transfections, each run in triplicate, were performed. One or two asterisks indicate statistical significance ( $<<0.05$ or $<0.01$, respectively). B) Analysis of STAT3 and c-Jun binding to the proximal section of the MMP-1 promoter by chromatin immunoprecipitation (ChIP). HT-29 cells were optionally treated with IL-6 (top panel) or TPA (bottom panel) as indicated and then subjected to ChIP. After crosslinking of protein to DNA, antibodies to STAT3 or c-Jun were employed for precipitation as indicated. Unspecific IgG served as isotype control. PCR products obtained with MMP-1 promoter-specific primers were separated through 8\% polyacrylamide gels. As input control, a sample not subjected to immunoprecipitation was employed. C) Effect of mutations within the twin STAT/AP-1 binding site on activation of the MMP-1 minimal promoter. Reporter gene constructs with inactivating point mutations in either of the STAT or AP-1 binding sites (bold letters in the boxed consensus elements) were stimulated and analyzed for induced luciferase activity as in A. D) Activation of STAT3 by specific stimuli of HT-29 cells. Cells were optionally stimulated with IL-6 or/and TPA as indicated. Cells were lysed and subjected to PAGE, Western blot and immunochemical detection by antibodies to STAT3 pTyr705 and STAT3.

action with both the isolated SBE and AP-1BE. c-Jun could interact in a stimulus-independent manner not only with the AP-1BE, but also with the SBE. Disruption of the SBE greatly reduced STAT3 binding. Interestingly, constitutive c-Jun binding was also widely lost, but a reduced level of TPA-induced binding to the mutated SBE was retained. Similarly, disruption of the AP-1 BE widely abolishes both c-Jun and pY-STAT3 binding with a notable exception that a minor level of c-Jun binding upon co-stimulation with IL-6 and TPA was preserved. We attribute this to a mutual inter- dependence of both factors with regard to their capability to interact with their respective recognition sequences. From these findings we infer that c-Jun binding to the MMP-1 promoter is stimulation-independent but modulated by STAT3 and a STAT recognition DNA element.

Because we were not able to show phospho-Jun binding to DNA by Western blot analysis (data not shown) and could not therefore evaluate activation-dependent contacts with STAT3, we strived to demonstrate the induction of interactions between the two transcription factors. Figure 1C shows 
that upon co-stimulation with IL-6 and TPA, c-Jun could be co-precipitated with pY-STAT3.

Combinatorial contributions of STAT3 and AP-1 to transcriptional regulation of a minimal MMP-1 promoter. A set of reporter gene experiments was performed using the minimal MMP-1 promoter construct pMMP-1 - $0.6 \mathrm{~kb}$ containing a twin STAT/AP-1 binding element roughly $70 \mathrm{bp}$ upstream of the transcriptional start site [16]. Stimulation of transfected cells with both IL- 6 and TPA yielded a significant increase in transcriptional activity, indicating that both STAT3 and AP-1 contribute by individual expressioninducing effects. Co-stimulation with IL-6 and TPA resulted in an additive effect, suggesting cooperative activities of both transcription factors (Figure 2A). Both pY-STAT3 and the AP-1 protein c-Jun stimulus-specifically interact with the proximal MMP-1 promoter fragment, since promoter sequences crosslinked to the respective transcription factors could be amplified by PCR in a chromatin immunoprecipitation experiment (Figure 2B).

Disruption of the STAT3 binding site does not eliminate stimulation by IL-6 (Figure 2C, left hand panel). The only difference to the wild type promoter fragment is that the relative activity of IL-6 compared to TPA is slightly reduced. The finding that IL- 6 and TPA still have an additive effect on transcription suggests that interaction of STAT3 with the promoter fragment is possible despite the absence of an intact STAT cognate element. In contrast, disruption of the AP-1 binding site diminishes the response to IL-6, indicating that STAT3 action is impeded. Interestingly, TPA can still induce a significant degree of stimulation, though to a reduced level as compared to the wild type situation, indicating that AP-1 can exert activity even in the absence of an integral binding site (Figure 2C). A double mutant construct yielded no significant response to neither stimulus (data not shown). We could exclude the possibility that residual TPA-induced transcriptional activity might result from a certain degree of TPA participation in the activation of STAT3 via signaling crosstalk, since no trace of TPA-induced Tyr-phosphorylation of STAT3 was observed (Figure 2D).

Repressive effect of STAT3 on AP-1 control of MMP-1 promoter activity in the genomic context. To complement data obtained using a minimal promoter-based reporter gene model system we analyzed by quantitative reverse transcription PCR the combined influence of STAT3 and AP-1 on MMP-1 expression driven by the natural promoter in a HT-29 cellular context (Figure 3A). The MMP-1 promoter, apart from additional STAT cognate elements [16], contains various potential recognition sequences for transcription factors $[19,24,25]$ which all potentially contribute to complex transcriptional regulation. While both IL- 6 and TPA stimulated increased MMP-1 mRNA levels, the combination of both did not show an additive effect comparable to that observed for reporter gene induction controlled by the minimal promoter. Unexpectedly, IL-6 rather reduced the degree of TPA-induced expression stimulation drasti- cally. These data strongly suggest that divergently from its effects on the minimal MMP-1 promoter, STAT3 activation dampens AP-1-mediated MMP-1 mRNA synthesis.

To further study this effect, we knocked down STAT3 expression in HT-29 cells by shRNA (Figure 3B) and analyzed effects of AP- 1 activation on MMP-1 promoter activity under these conditions. As expected, IL-6-dependent induction of MMP-1 mRNA was reduced upon STAT3 knock-down. In contrast, transcriptional activation in response to stimulation by both TPA and the combination of IL- 6 and TPA stimulation was significantly enhanced, supporting the notion that STAT3 has a dampening effect on AP-1-driven MMP-1 expression in HT-29 CRC cells (Figure 3C).

Functional interference of STAT3 and STAT1 in transcriptional regulation of the MMP-1 promoter. This unexpected finding prompted us to strive for a more complete picture of STAT effects on MMP-1 promoter activity in CRC. Previously, we have observed that elevated STAT1 activity, both in absolute terms and in relation to STAT3 activity, is correlated with favorable prognosis in colorectal cancer. Moreover, we could demonstrate the occurrence of DNA binding STAT1/STAT3 heterodimers in the majority of CRC biopsies [23]. We therefore included STAT1 activation in our analysis of MMP-1 promoter control in HT-29 cells and first established interferon- $\gamma($ IFN- $\gamma)$ as a highly specific stimulus for STAT1 tyrosine phosphorylation (Figure 4A). Unlike IL- 6 and TPA, IFN- $\gamma$ did not induce significant stimulation of MMP-1 mRNA biosynthesis. However, it elicited an interesting influence when applied in combination with IL- 6 and/ or TPA (Figure 4B). While IFN- $\gamma$ tendentially repressed both IL-6 and TPA stimuli, it showed a striking super-additive, synergistic effect on MMP-1 expression in a triple combination with both IL-6 and TPA.

STAT3 knock-down was again employed to more thoroughly characterize the surprising contribution of STAT1 to the AP-1 and STAT3 mediated control of MMP-1 transcription in HT-29 cells (Figure 4C). In line with Figure 3B, upon treatment with STAT3 shRNA, the suppressive effect of STAT3 on TPA-driven transcriptional activation of the MMP-1 gene is lost and respective stimulation indices rise. In contrast, the synergistic effect of IFN- $\gamma$ stimulation is significantly reduced in the absence of STAT3.

Finally we used a stable STAT3 k.o derivative of HT-29 to address the consequences of STAT3 deprivation for STAT1 activation. Interestingly, STAT3 knock-down was found to render STAT1 susceptible to a limited but significant level of IL-6-dependent activation (Figure 4D). This result suggests that in HT-29 cells STAT1 activation in response to IL-6 is repressed by STAT3 activity.

\section{Discussion}

We have characterized mutual functional connections of transcription factors STAT3, STAT1, and AP-1 in activity control of the MMP-1 promoter in colorectal carcinoma cells. 
A

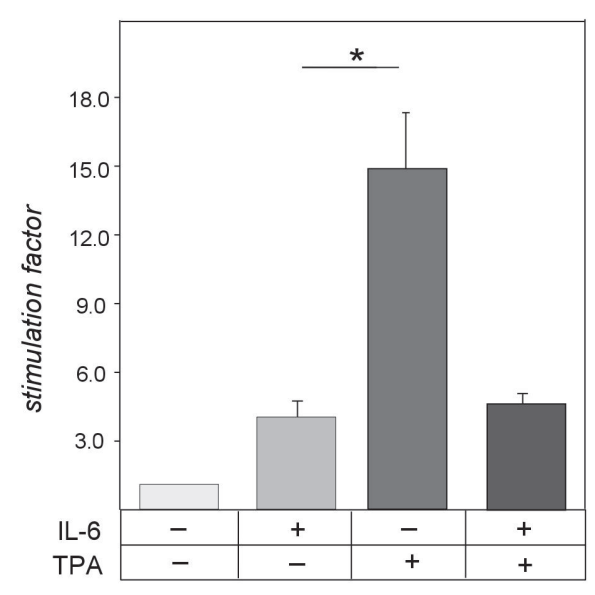

B

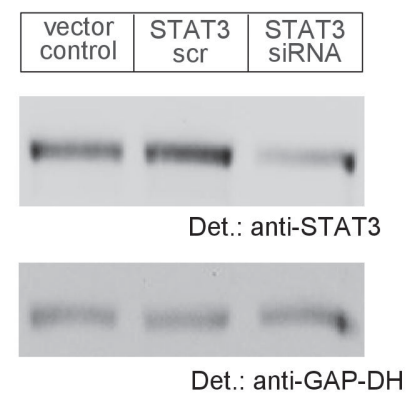

C

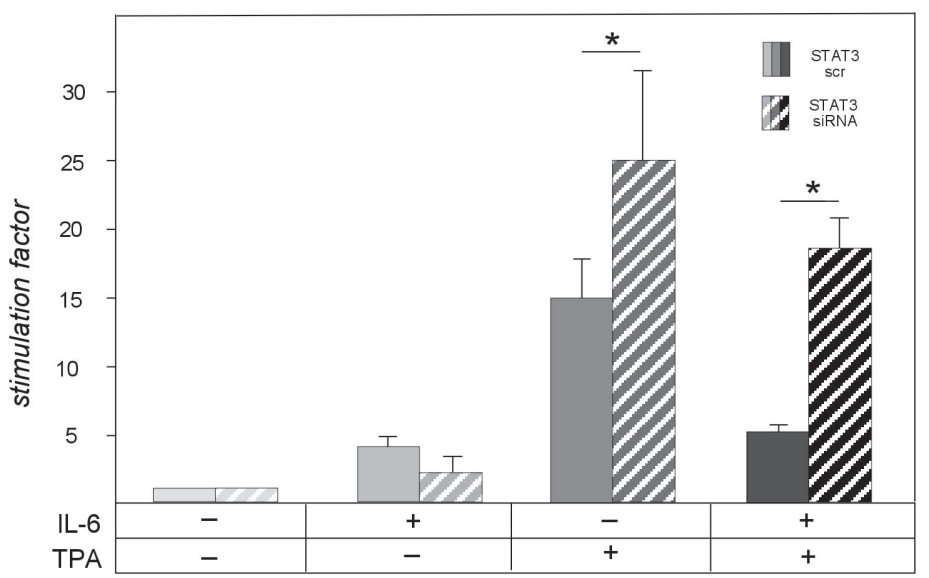

Figure 3. Effect of external stimuli on expression of the endogenous MMP-1 gene in HT-29 cells. A) Relative induction of MMP- 1 mRNA levels in HT-29 cells in response to stimulation with IL-6 or/and TPA. From HT-29 cells incubated with the indicted stimuli for $12 \mathrm{~h}$, cDNA was prepared, total RNA was isolated and reverse transcribed and subjected to quantitative PCR using specific primer pairs for MMP- 1 and $\beta$-actin. The determined copy number of MMP-1 mRNA was normalized to $\beta$-actin mRNA. Stimulation factors are expressed as relative changes of MMP-1 mRNA copy numbers under the indicated stimulation conditions compared to non-stimulated cells. Data represent the means of three independent experiments. An asterisk indicates statistical significance $(\mathbf{p}<0.05)$ determined by Student's t-test. B) siRNA-mediated knock-down of STAT3 in HT-29 cells. HT-29 cells transfected with plasmid pSUPERneoSTAT3si encoding a STAT3-specific RNAi sequence or, as control, with the empty pSUPERneo were lysed and subjected to Western blot detection using an anti-STAT3 antibody. Equal loading of lanes was verified by re-probing for GAPDH. C) Effect of STAT3 knock-down on stimulus-dependent changes in MMP-1 mRNA levels in HT-29 cells. $96 \mathrm{~h}$ post transfection, cells transfected with pSUPERneoSTAT3scr (control, filled bars) and pSUPERneoSTAT3si (striped bars) were stimulated with IL-6 or/and TPA as indicated and analyzed for MMP-1 mRNA contents and respective stimulation factors by quantitative RT-PCR as in A.

Analyzing transcription factor interplay in the context of a minimal promoter, we were able to co-precipitate c-Jun with pY-STAT3 upon co-stimulation of cells with IL-6 and TPA. This finding is in line with the established potency of STAT3 to immediately interact with c-Jun [26] and to modulate promoter activity by means of direct protein-protein contacts with other transcription factions, e.g. with NF-kB [27].
The notion of combined STAT3 and AP-1 involvement in MMP-1 promoter regulation has also been supported by DNA-protein complex formation experiments we performed in the context of an earlier study [16]. In this paper, we showed the appearance of an assembly on the combined STAT3/AP-1 binding element which became impaired when the STAT3 binding element was disrupted. 
A

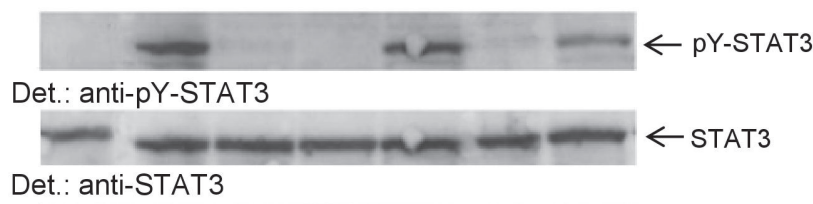

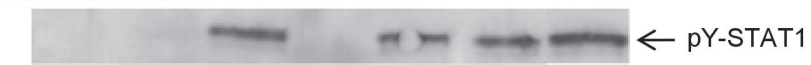

Det.: anti-pY-STAT1

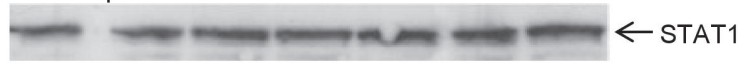

Det.: anti-STAT1

\begin{tabular}{l|c|c|c|c|c|c|c|}
\cline { 2 - 7 } IL-6 & - & + & - & - & + & - & + \\
\cline { 2 - 8 } IFN- $\gamma$ & - & - & + & - & + & + & + \\
\cline { 2 - 8 } TPA & - & - & - & + & - & + & + \\
\cline { 2 - 8 } & & &
\end{tabular}

B

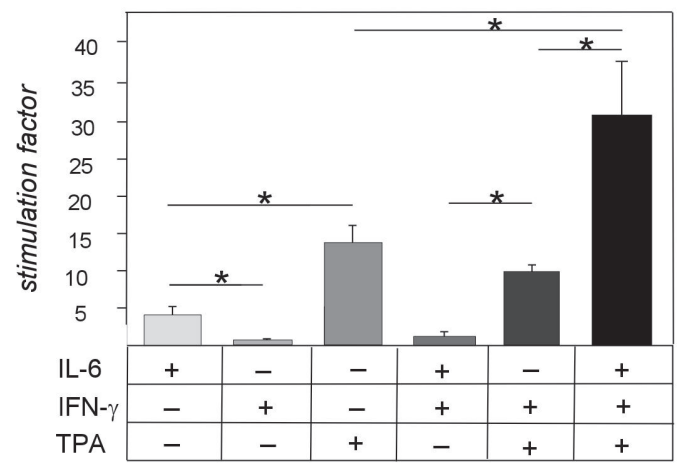

C

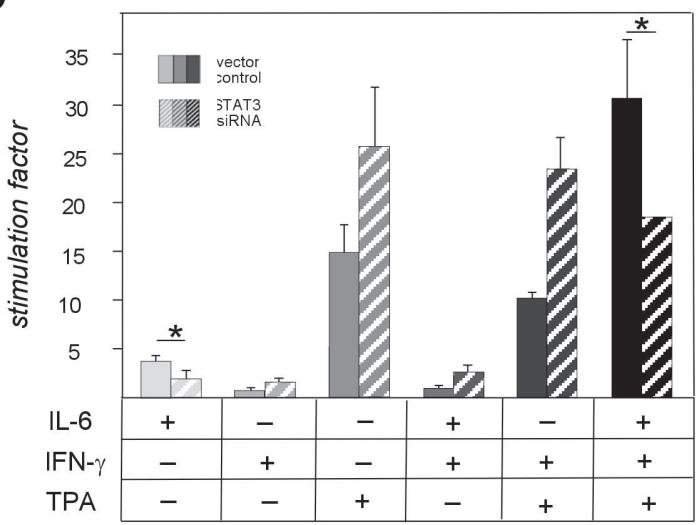

D

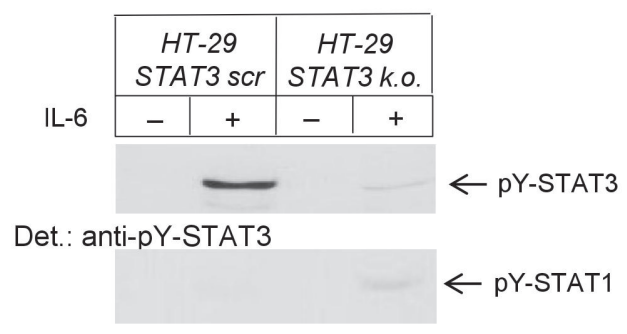

Det.: anti-pY-STAT1

$\leftarrow \beta$-actin

Det.: anti- $\beta$-actin

Figure 4. Interference of STAT1 activation with the functions of STAT3 and AP-1 in MMP-1 regulation in HT-29 cells. A) Activation of STAT1 versus STAT3 by specific stimuli of HT-29 cells. Samples of $2 \times 10^{5}$ HT- 29 cells were optionally stimulated with IL- 6 or/and TPA and/or IFN- $\gamma$ for 30 min as indicated and analyzed as in Figure 1D. Western Blots were probed with antibodies to (phosho-) STAT3 and (phosho-) STAT1 as indicated. B) Relative induction of MMP-1 mRNA in HT-29 cells in response to stimulation with IL-6/TPA/IFN- $\gamma$. HT-29 cells were incubated with the indicated stimuli individually or in combination as in A. Quantification and presentation of relative MMP-1 mRNA levels was as in Figure 3A. C) Effect of STAT3 knock-down on MMP-1 mRNA levels induced by individual and combined activation of STAT3, AP-1 and STAT1. HT-29 cells optionally subjected to siRNA-mediated STAT3 depletion as in Figure 3C were treated with the indicated stimuli. Quantification and presentation of relative MMP-1 mRNA levels was performed as in Figure 3B. Filled bars represent stimulation factors determined for control (vector transfected) cells, striped bars depict factors obtained for STAT3 k.o. cells. Three independent experiments were performed, asterisks indicate statistical significance of differences ( $\mathbf{p}<0.05$ ). D) Effect of stable STAT3 depletion on STAT1 activation in HT-29 cells. STAT3 knock-down in HT-29 cells carrying a stable knock-down of STAT3 (HT-29 STAT3 k.o.) and control cells stably transduced with a scrambled STAT3 shRNA (HT-29 STAT3 scr) were stimulated with IL-6 as indicated and analyzed by Western blot detection for STAT3 and STAT1 activation (tyrosine phosphorylation) as in A. Equal loading was verified by re-probing for $\beta$-actin.

The experiments presented here are in line with the observation that aberrant activities of STAT3 and AP-1 correlate in colorectal carcinoma biopsies [16]. It is not clear, however, how activities of both transcription combine in regulating malignancy-related genes in different types of cells.

The MMP-1 minimal promoter and the isolated proximal STAT/AP-1 twin cognate element behave such that stimulatory effects on transcription are additive in first approximation in HT-29 cells. We have recently obtained evidence for a similar tendency in the lung cancer cell line A549 [28].

In HT-29 cells, a more complex situation emerged in the context of full length MMP-1 promoter or if the natural genomic setting was concerned. Under these conditions,
STAT3 activation was observed to dampen AP-1-mediated MMP-1 mRNA synthesis instead of enhancing it. Various consensus recognition elements for STAT factors, AP-1, GATA binding and ETS proteins are present in the genomic DNA sequence upstream of the MMP-1 transcriptional start site [24, 25]. Interestingly, it has also been reported recently that in melanoma cells, TPA can activate several tyrosine phosphatases which dephosphorylate pTyr-STAT3 [29]. Related mechanisms may to some extent explain why in HT-29 CRC cells TPA appears to reduce IL-6-induced STAT3 phosphorylation (Figure 2D). However, this effect is obviously functionally overridden by the dampening influence of IL- 6 on TPA-driven MMP-1 promoter activation. 
Moreover, STAT1 exerted a profound effect on MMP-1 promoter activity (perhaps through hetero-dimerization with STAT3 or/and by regulatory influence through contact with existing STAT binding sites within the extended MMP-1 promoter region) which becomes particularly pronounced in the absence of (activated) STAT3. We interpret these findings such that in HT-29 cells, STAT3 has a repressive influence on STAT1 function.

The noticeable involvement of STAT1 in interdependence with AP-1 may also be an indication of cooperation between these two factors in the MMP-1 transcription regulation. Such crosstalk was described for the nitric oxide synthase (NOS) 2 and intercellular adhesion molecule-1 promoters which both contain adjacent STAT and AP-1 recognition elements. They show enhanced interferon- $\gamma$-induced stimulation through combined activities of STAT1 and c-Fos or SP-1, respectively [30, 31].

Recent findings by our group [17] imply that the cellular context is decisive for the consequences of the STAT3/STAT1 interplay in CRC cell lines with regard to malignancy and in vivo tumor growth. While in HT-29 cells the established tumor suppressive function of STAT1 $[9,10,32]$ is overridden by STAT3, in other CRC cell lines such as HCT116 and SW620 this is obviously not the case [17]. We have, thus, put forward the concept that the balance of STAT3 and STAT1 activity in CRC influences cancer properties and development [18]. Since we already showed that the ratio of STAT3 and STAT1 expression and/or activity in biopsies from CRC patients is of prognostic relevance [17], it will be very interesting to investigate in the future if inclusion of AP- 1 activity into the analysis can add further predictive value.

Acknowledgements: This work was supported by a scholarship of the "Interdisziplinäres Zentrum für Klinische Forschung (IZKF)" of the Jena University Hospital to A.M. and by a grant by the German Federal Ministry of Education and Research, grant 01DH16014 to M.K. and K.F. We thank Dieter Kube for the STAT3 shRNA construct.

\section{References}

[1] DECLAN FLEMING RY. Colorectal cancer screening and follow-up. Surg Oncol 1998; 7: 125-137.

[2] OVING IM, CLEVERS HC. Molecular causes of colon cancer. Eur J Clin Invest 2002; 32: 448-457.

[3] VAIOPOULOS AG, PAPACHRONI KK, PAPAVASSILIOU AG. Colon carcinogenesis: Learning from NF-карраB and AP-1. Int J Biochem Cell Biol 2010; 42: 1061-1065. https:// doi.org/10.1016/j.biocel.2010.03.018

[4] KLAMPFER L. The role of signal transducers and activators of transcription in colon cancer. Front Biosci 2008; 1: 28882899.

[5] CORVINUS FM, ORTH C, MORIGGL R, TSAREVA SA, WAGNER $S$ et al. Persistent STAT3 activation in colon cancer is associated with enhanced cell proliferation and tumor growth. Neoplasia 2005; 7: 545-555.
[6] TSAREVA SA, MORIGGL R, CORVINUS FM, WIEDERANDERS B, SCHUTZ A et al. Signal transducer and activator of transcription 3 activation promotes invasive growth of colon carcinomas through matrix metalloproteinase induction. Neoplasia 2007; 9: 279-291.

[7] XIONG H, ZHANG ZG, TIAN XQ, SUN DF, LIANG QC et al. Inhibition of JAK1, 2/STAT3 signaling induces apoptosis, cell cycle arrest, and reduces tumor cell invasion in colorectal cancer cells. Neoplasia 2008; 10: 287-297.

[8] XIONG H, HONG J, DU W, LIN YW, REN LL et al. Roles of STAT3 and ZEB1 proteins in E-cadherin down-regulation and human colorectal cancer epithelial-mesenchymal transition. J Biol Chem 2012; 287: 5819-5832. https://doi. org/10.1074/jbc.M111.295964

[9] WIDSCHWENDTER A, TONKO-GEYMAYER S, WELTE T, DAXENBICHLER G, MARTH C et al. Prognostic significance of signal transducer and activator of transcription 1 activation in breast cancer. Clin Cancer Res 2002; 8: 30653074.

[10] SIMPSON JA, AL-ATTAR A, WATSON NF, SCHOLEFIELD JH, ILYAS $M$ et al. Intratumoral $\mathrm{T}$ cell infiltration, MHC class I and STAT1 as biomarkers of good prognosis in colorectal cancer. Gut 2010; 59: 926-933. https://doi. org/10.1136/gut.2009.194472

[11] OSSOWSKI L. Invasion of connective tissue by human carcinoma cell lines: requirement for urokinase, urokinase receptor, and interstitial collagenase. Cancer Res 1992; 52: 6754-6760.

[12] ITOH M, MURATA T, SUZUKI T, SHINDOH M, NAKAJIMA K et al. Requirement of STAT3 activation for maximal collagenase-1 (MMP-1) induction by epidermal growth factor and malignant characteristics in T24 bladder cancer cells. Oncogene 2006; 25: 1195-1204. https://doi.org/10.1038/ sj.onc. 1209149

[13] MURRAY GI, DUNCAN ME, O'NEIL P, MELVIN WT, FOTHERGILL JE. Matrix metalloproteinase-1 is associated with poor prognosis in colorectal cancer. Nat Med 1996; 2: 461-462.

[14] BENDARDAF R, LAMLUM H, VIHINEN P, RISTAMAKI $\mathrm{R}$, LAINE $\mathrm{J}$ et al. Low collagenase-1 (MMP-1) and MT1MMP expression levels are favourable survival markers in advanced colorectal carcinoma. Oncology 2003; 65: 337346. https://doi.org/10.1159/000074647

[15] SHIOZAWA J, ITO M, NAKAYAMA T, NAKASHIMA M, KOHNO S et al. Expression of matrix metalloproteinase-1 in human colorectal carcinoma. Mod Pathol 2000; 13: 925-933. https://doi.org/10.1038/modpathol.3880169

[16] ZUGOWSKI C, LIEDER F, MÜLLER A, GASCH J, CORVINUS FM et al. STAT3 controls matrix metalloproteinase-1 expression in colon carcinoma cells by both direct and AP-1-mediated interaction with the MMP-1 promoter. Biol Chem 2011; 392: 449-459. https://doi.org/10.1515/ BC.2011.038

[17] NIVARTHI H, GORDZIEL C, THEMANNS M, KRAMER $\mathrm{N}$, EBERL $\mathrm{M}$ et al. The ratio of STAT1 to STAT3 expression is a determinant of colorectal cancer growth. Oncotarget 2016; 7: 51096-51106. https://doi.org/10.18632/oncotarget.9315 
[18] FRIEDRICH K, DOLZNIG H, HAN X, MORIGGL R. Steering of carcinoma progression by the YIN/YANG interaction of STAT1/STAT3. Biosci Trends 2017; 11: 1-8. https://doi. org/10.5582/bst.2016.01250

[19] RUTTER JL, MITCHELL TI, BUTTICÈ G, MEYERS J, GUSELLA JF et al. A single nucleotide polymorphism in the matrix metalloproteinase-1 promoter creates an Ets binding site and augments transcription. Cancer Res 1998; 58: 5321-5325.

[20] HOLTICK U, VOCKERODT M, PINKERT D, SCHOOF N, STÜRZENHOFECKER B et al. STAT3 is essential for Hodgkin lymphoma cell proliferation and is a target of tyrphostin AG17 which confers sensitization for apoptosis. Leukemia 2005; 19: 936-944. https://doi.org/10.1038/sj.leu.2403750

[21] WOHLMANN A, SEBASTIAN K, BOROWSKI A, KRAUSE S, FRIEDRICH K. Signal transduction by the atopy-associated human thymic stromal lymphopoietin (TSLP) receptor depends on Janus kinase function. Biol Chem 2010; 391: 181-186. https://doi.org/10.1515/BC.2010.029

[22] BARUCKER C, HARMEIER A, WEISKE J, FAULER B, ALBRING KF et al. Nuclear translocation uncovers the amyloid peptide $\mathrm{A} \beta 42$ as a regulator of gene transcription. J Biol Chem 2014; 289: 20182-20191. https://doi.org/10.1074/jbc. M114.564690

[23] GORDZIEL C, BRATSCH J, MORIGGL R, KNÖSEL T, FRIEDRICH K. Both STAT1 and STAT3 are favourable prognostic determinants in colorectal carcinoma. Br J Cancer 2013; 109: 138-146. https://doi.org/10.1038/bjc.2013.274

[24] BIDDER M, LOEWY AP, LATIFI T, NEWBERRY EP, FERGUSON G et al. Ets domain transcription factor PE1 suppresses human interstitial collagenase promoter activity by antagonizing protein-DNA interactions at a critical AP1 element. Biochemistry 2000; 39: 8917-8928.

[25] BEHERA AK, THORPE CM, KIDDER JM, SMITH W, HILDEBRAND E et al. Borrelia burgdorferi-induced expression of matrix metalloproteinases from human chondrocytes requires mitogen-activated protein kinase and janus kinase/ signal transducer and activator of transcription signaling pathways. Infect. Immun 2004; 72: 2864-2871.
[26] GINSBERG M, CZEKO E, MÜLLER P, REN Z, CHEN X et al. Amino acid residues required for physical and cooperative transcriptional interaction of STAT3 and AP-1 proteins c-Jun and c-Fos. Mol Cell Biol 2007; 18: 6300-6308. https:// doi.org/10.1128/MCB.00613-07

[27] YANG J, LIAO X, AGARWAL MK, BARNES L, AURON PE et al. Unphosphorylated STAT3 accumulates in response to IL-6 and activates transcription by binding to NF kappa B. Genes Dev 2007; 21: 1396-1408. https://doi.org/10.1101/ gad.1553707

[28] SCHÜTZ A, RÖSER K, KLITZSCH J, LIEDER F, ABERGER $F$ et al. Lung adenocarcinomas and lung cancer cell lines show association of MMP-1 expression with STAT3 activation. Transl Oncol 2015; 8: 97-105. https://doi.org/10.1016/j. tranon.2015.02.002

[29] Oka M, Sumita N, Sakaguchi M, Iwasaki T, Bito T et al. 12-O-tetradecanoylphorbol-13-acetate inhibits melanoma growth by inactivation of STAT3 through protein kinase Cactivated tyrosine phosphatase(s). J Biol Chem 2009; 284: 30416-30423. https://doi.org/10.1074/jbc.M109.001073

[30] XU W, COMHAIR AAS, ZHENG S, CHU SC, MARKSKONCZALIK J et al. STAT-1 and c-Fos interaction in nitric oxide synthase-2 gene activation. Am J Physiol Lung Cell Mol Physiol 2003; 285: 137-148. https://doi.org/10.1152/ajplung.00441.2002

[31] LOOK DC, PELLETIER MR, TIDWELL RM, ROSWIT WT, HOLTZMAN MJ. Stat1 depends on transcriptional synergy with Sp1. J Biol Chem 1995; 270: 30264-30267.

[32] HANADA T, KOBAYASHI T, CHINEN T, SAEKI K, TAKA$\mathrm{KI} \mathrm{H}$ et al. IFN gamma-dependent, spontaneous development of colorectal carcinomas in SOCS1-deficient mice. J Exp Med 2006; 203: 1391-1397. https://doi.org/10.1084/ jem.20060436 